\title{
Performance of Personal Workspace Controls Final Report
}

\author{
Francis Rubinstein \\ Sila Kiliccote \\ John Loffeld \\ Lawrence Berkeley National Laboratory \\ Pete Pettler \\ Joel Snook \\ Vistron
}

This work was supported by the Assistant Secretary for Energy Efficiency and Renewable Energy, Building Technologies Program, of the U.S. Department of Energy under Contract No. DE-AC03-76SF00098. 


\section{Introduction}

One of the key deliverables for the DOE-funded controls research at LBNL for FY04 was the development of a prototype Personal Workspace Control system. The successful development of this system is a critical milestone for the LBNL Lighting Controls Research effort because this system demonstrates how IBECS can add value to today's Task Ambient lighting systems. LBNL has argued that by providing both the occupant and the facilities manager with the ability to precisely control the operation of overhead lighting and all task lighting in a coordinated manner, that task ambient lighting can optimize energy performance and occupant comfort simultaneously [Reference Task Ambient Foundation Document]. The Personal Workspace Control system is the application of IBECS to this important lighting problem.

This report discusses the development of the Personal Workspace Control to date including descriptions of the different fixture types that have been converted to IBECS operation and a detailed description of the operation of PWC Scene Controller, which provides the end user with precise control of his task ambient lighting system.

\section{Project Objectives}

The objective, from the Annual Plan, is:

Demonstrate improvements in efficiency, lighting quality and occupant comfort realized using Personal Workspace Controls (PWC) designed to optimize the delivery of lighting to the individual's workstation regardless of which task-ambient lighting solution is chosen. The PWC will be capable of controlling floor-mounted, desk lamps, furniture-mounted and overhead lighting fixtures from a personal computer and handheld remote. The PWC will use an environmental sensor to automatically monitor illuminance, temperature and occupancy and to appropriately modulate ambient lighting according to daylight availability and to switch off task lighting according to local occupancy. [Adding occupancy control to the system would blunt the historical criticism of occupant-controlled lighting - the tendency of the occupant to leave lights on]. The PWC will be an entirely open networking system in which all manufacturers of task lights, furniture-mounted lighting and ballast manufacturers can participate and have a business position.

\section{Accomplishments to Date}

As of September 2004, LBNL has successfully accomplished most of the above objectives. Specifically, LBNL has 1) developed working prototypes of several different types of task lights and overhead lights that are easily controllable via IBECS and 2) completed a Scene Controller control panel that lets the user create a series of "presets" that each implement a different lighting scene. The remaining objectives as stated above will be implemented before December, 2004. 


\section{Overview of Task-Ambient Lighting Systems}

The office of tomorrow will boast not one, but several, lighting "systems." Ambient lighting for circulation and easy visual tasks will continue to be provided by overhead lights. The trend is toward direct/indirect lighting systems for ambient lighting since the lighting quality is higher than for direct systems. However, the power density for ambient lighting is under constant downwards pressure because of building codes, such as CA's Title 24 and ASHRAE 1999. For purposes of demonstration, LBNL has converted several direct indirect fixtures at LBNL so that they are controllable via IBECS. This is shown in the figure below.

Task lighting, which can be provided by a number of methods, is excellent for administering additional illumination where needed within the workstation. Task lighting falls into four categories:

1. Furniture-mounted lighting. This is lighting that is usually built-in to the furniture. Frequently, a luminaire will be mounted below a storage cabinet providing illumination where the overhead light may not reach.

2. $\quad$ Desk lamps. Many manufacturers make small desk lamps that are intended for placement on the desk surface or otherwise attached to the desk.

3. Table lamps. To add an aura of informality, residential table lamps are sometimes used in place of commercial desk lamps.

4. Floor-standing lighting. Floor-standing torchieres that use CFL lamps are becoming increasingly popular and inexpensive for commercial offices.

\section{Setting the Scene Controller}

For example, the user could assign an undercounter light at medium intensity, the overhead light to low intensity and a third floor-standing light to low intensity to a preset labeled "computer work". When the user select that button from their computer control panel, the lights immediately dim to the levels that the user has assigned to that preset. The user can create additional presets (say "full on" and "all off”) that would invoke those scenes when pressed. 


\section{The Personal Workspace Control System}

The Personal Workspace Control has been installed in two spaces at LBNL. The first of these spaces is a small office with an undercounter task light and a 3-way CFL-based table lamp. The Scene Controller control panel is configured so that this occupant can call four presets from his PC: Full, OFF, low and medium. Each preset is configured to recall a different lighting scene.
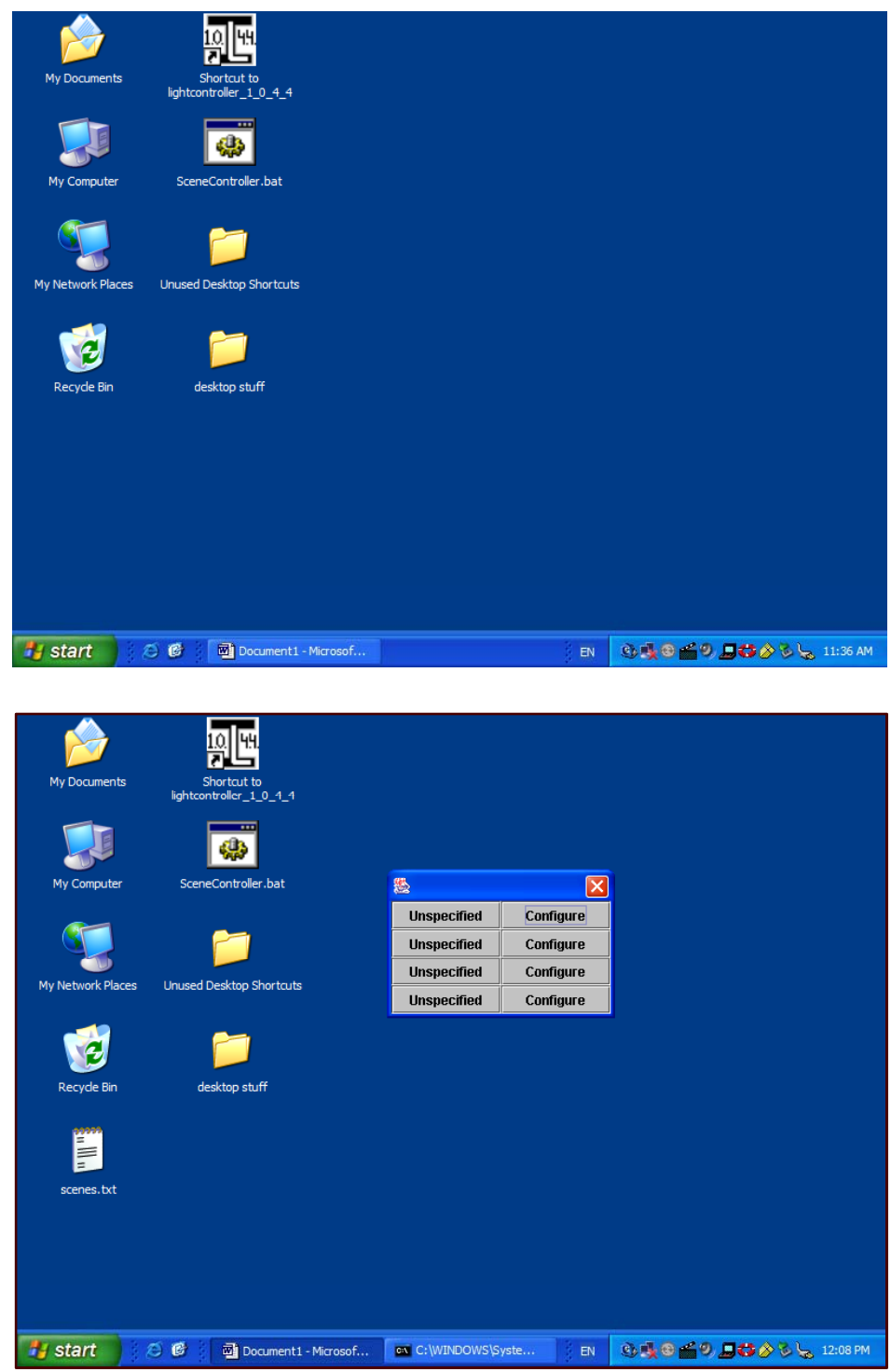

PC screen showing location of SceneController control panel. To start the Scene Controller, the user clicks on the icon labeled "Scene Controller.bat”.

This opens the Scene Controller control panel. Since the Scene Controller has not been set, all scenes are labeled "Unspecified”. 

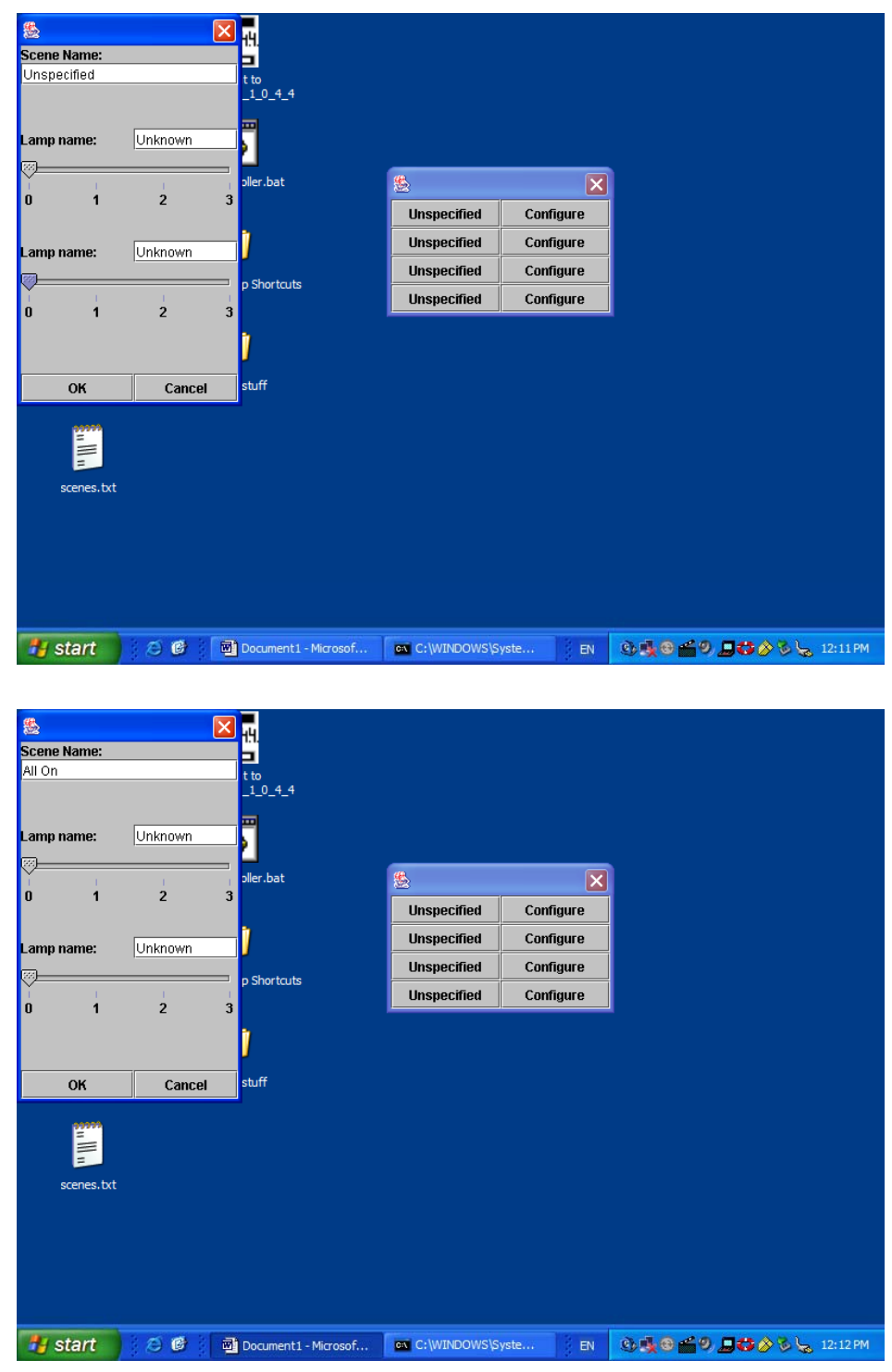

To configure the topmost preset button, the user clicks on the configure button immediately to the right of the button to be set. This opens up the configure panel for this scene. Note that the scene name is not set initially. Note also that two lamps are also unnamed.

To name the first scene, the user changes the top edit field to read “All On”. 

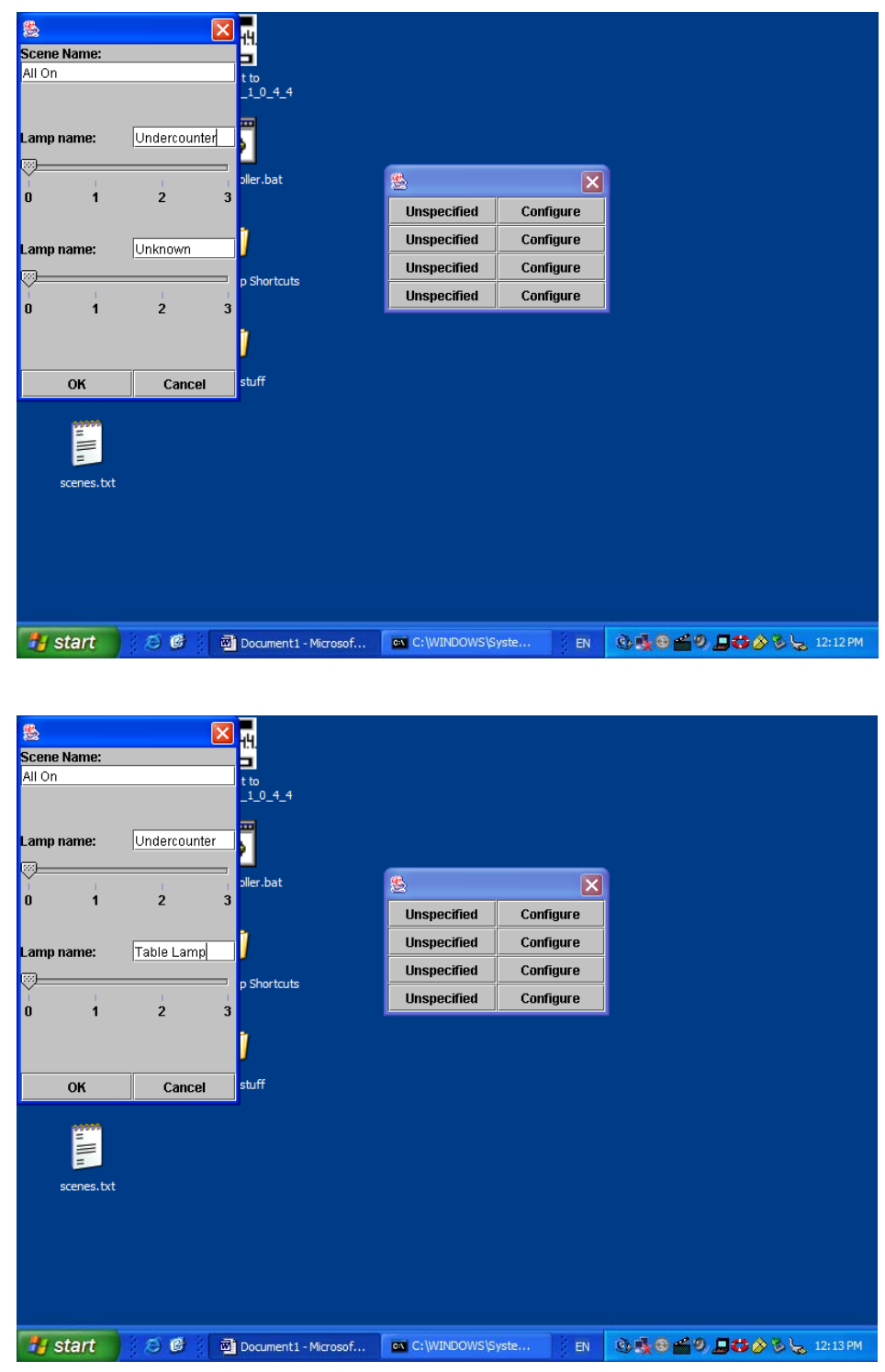

The first listed lamp corresponds to the undercounter light. So the user changes the name of the edit field accordingly.

Then the user labels the second light “Table Lamp”. The user then adjusts each slider to correspond to the desired fixture light level. In this case " 0 " corresponds to $\mathrm{ON}$. Then the user confirms the settings by clicking $\mathrm{OK}$. 


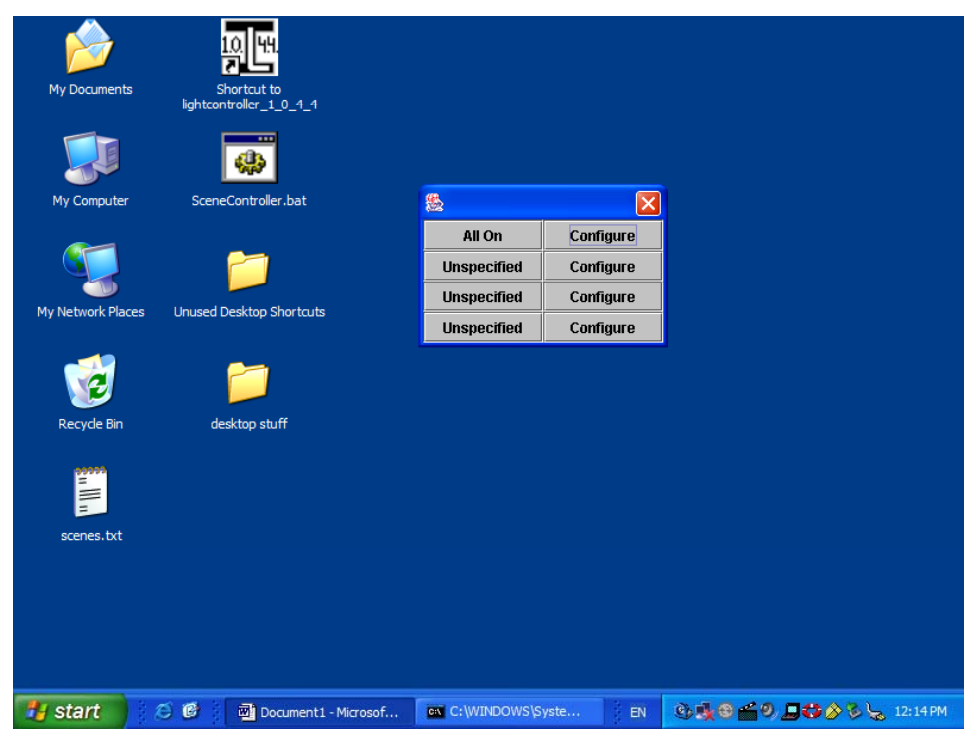

The configure window disappears and displays the updated control panel with the first preset set to "All On”.

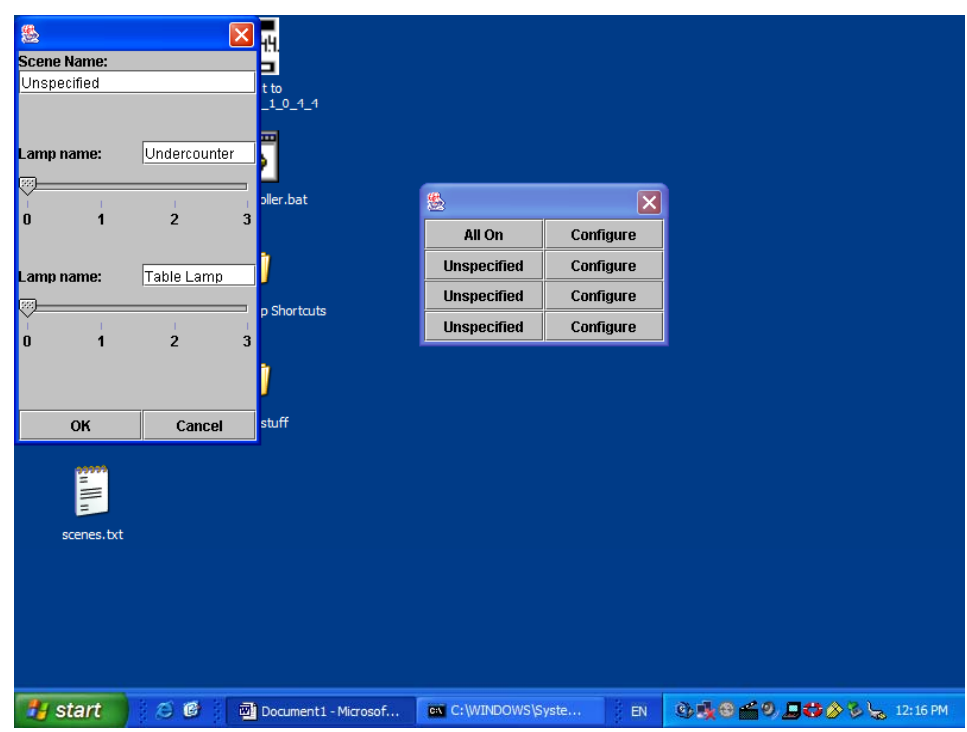

Now the user configures the second preset by clicking the configure button to the right of the button. The opens up the configure dialog box for the second preset. 

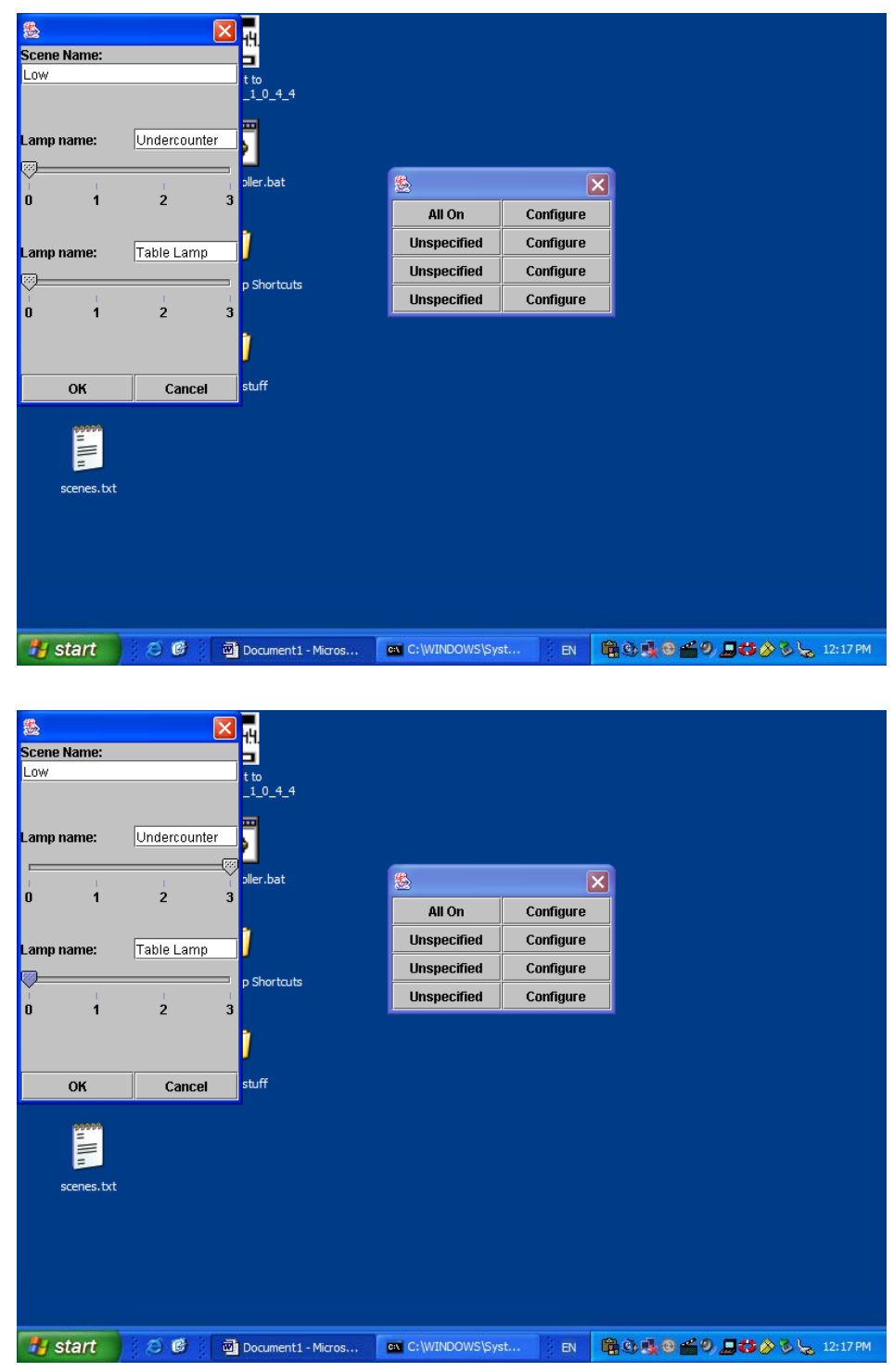

This time the user makes the Scene Name be "low" by editing the top field. Note that this time the lamp names are already filled in since the program "remembers" the lamp labels from the previous configure.

The user configures the Undercounter lamp to produce low light level by sliding the arrow to " 3 ". 

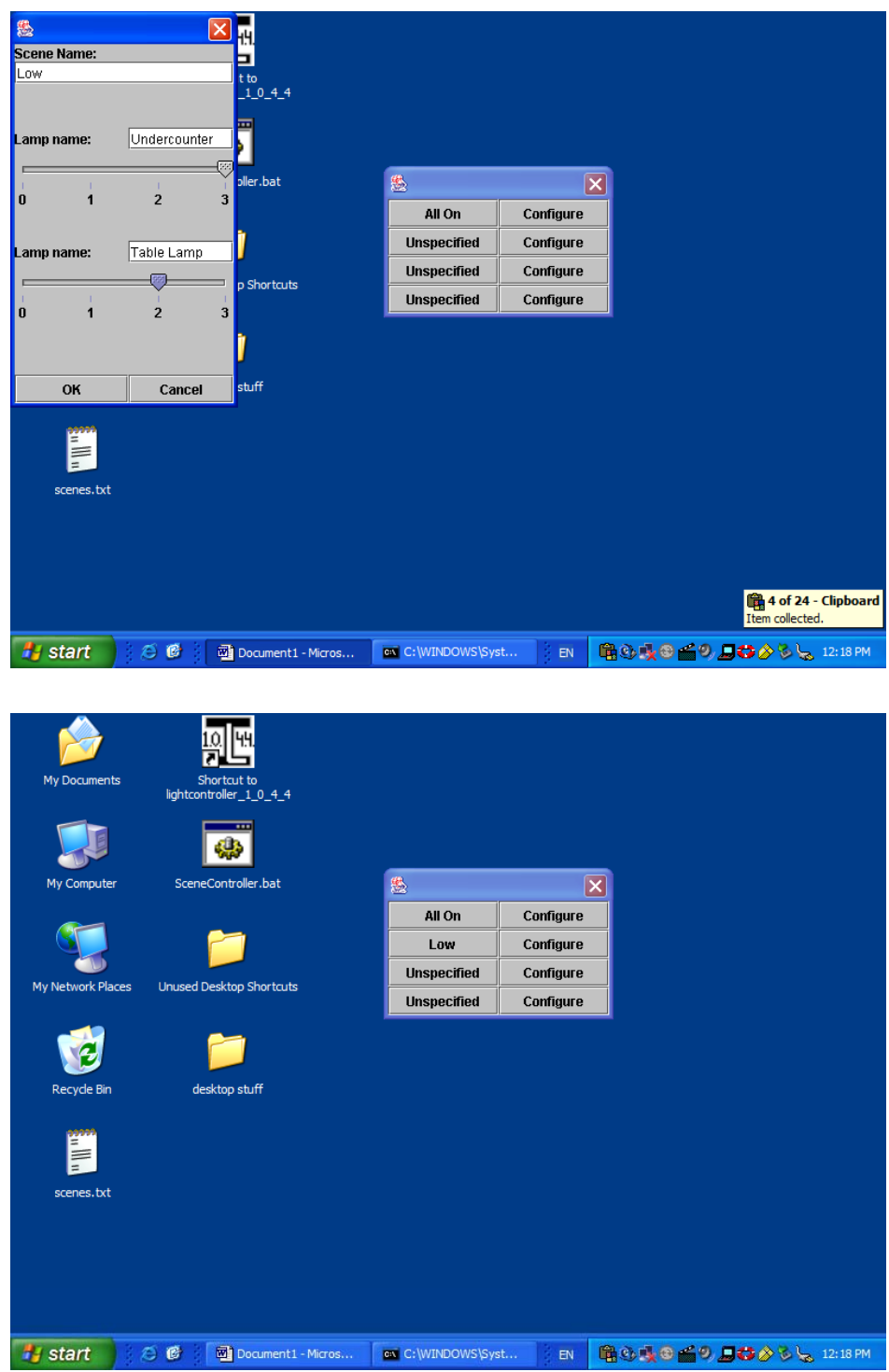

Similarly, the user sets the Table Lamp to the low setting ("2" in this case). The user then fixes this Low preset by clicking $\mathrm{OK}$.

This is updated Scene Controller control panel with the first two preset configured. Now when the user clicks the "All On” preset both lights immediately go that preset level (see Figure $\mathrm{x}$ ). When the user clicks "low", the lights go to low level (see figure y). 

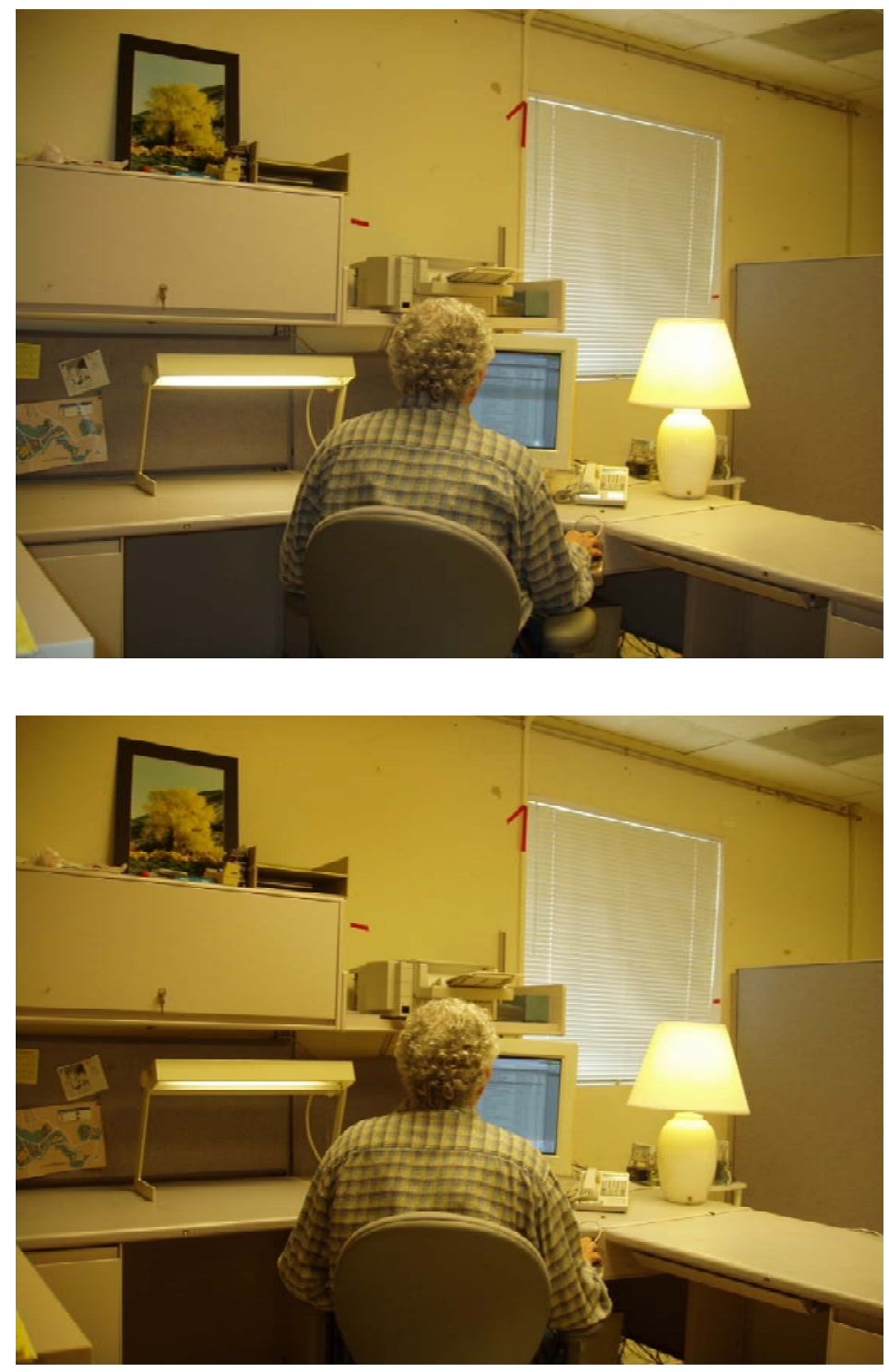

The table lamp (right) and undercounter light (left) are shown here both set to the All On preset.

The table lamp (right) and undercounter light (left) are shown here both set to the Low preset. 

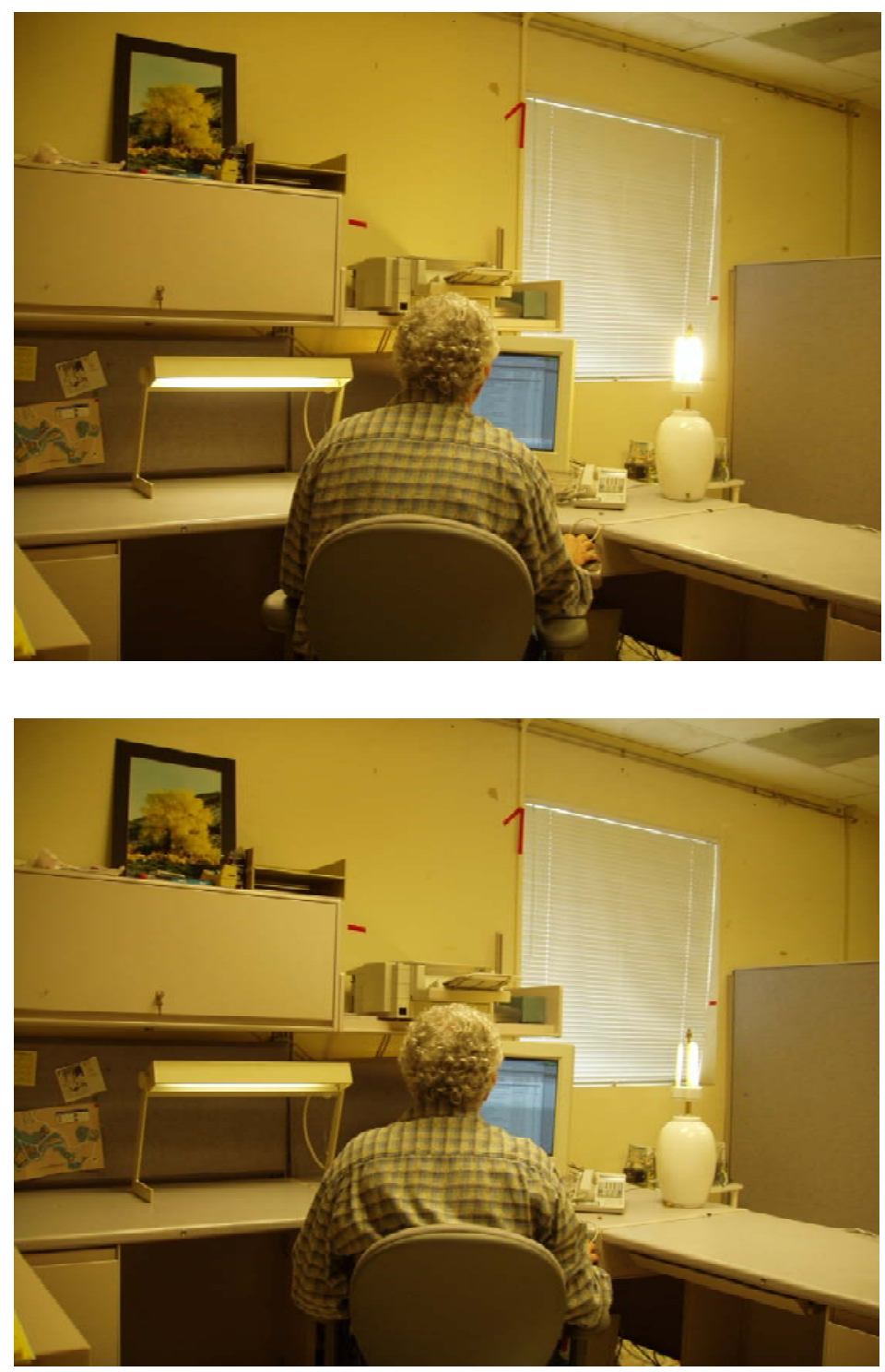

Scene Controller set to "All On” with shade removed from Table Lamp to show all three lamps are lit.

Scene Controller set to "Low" with shade removed from Table Lamp to show only one lamp is lit. Also Undercounter lamp is at low level (compare to Figure $\mathrm{x}$ ). 


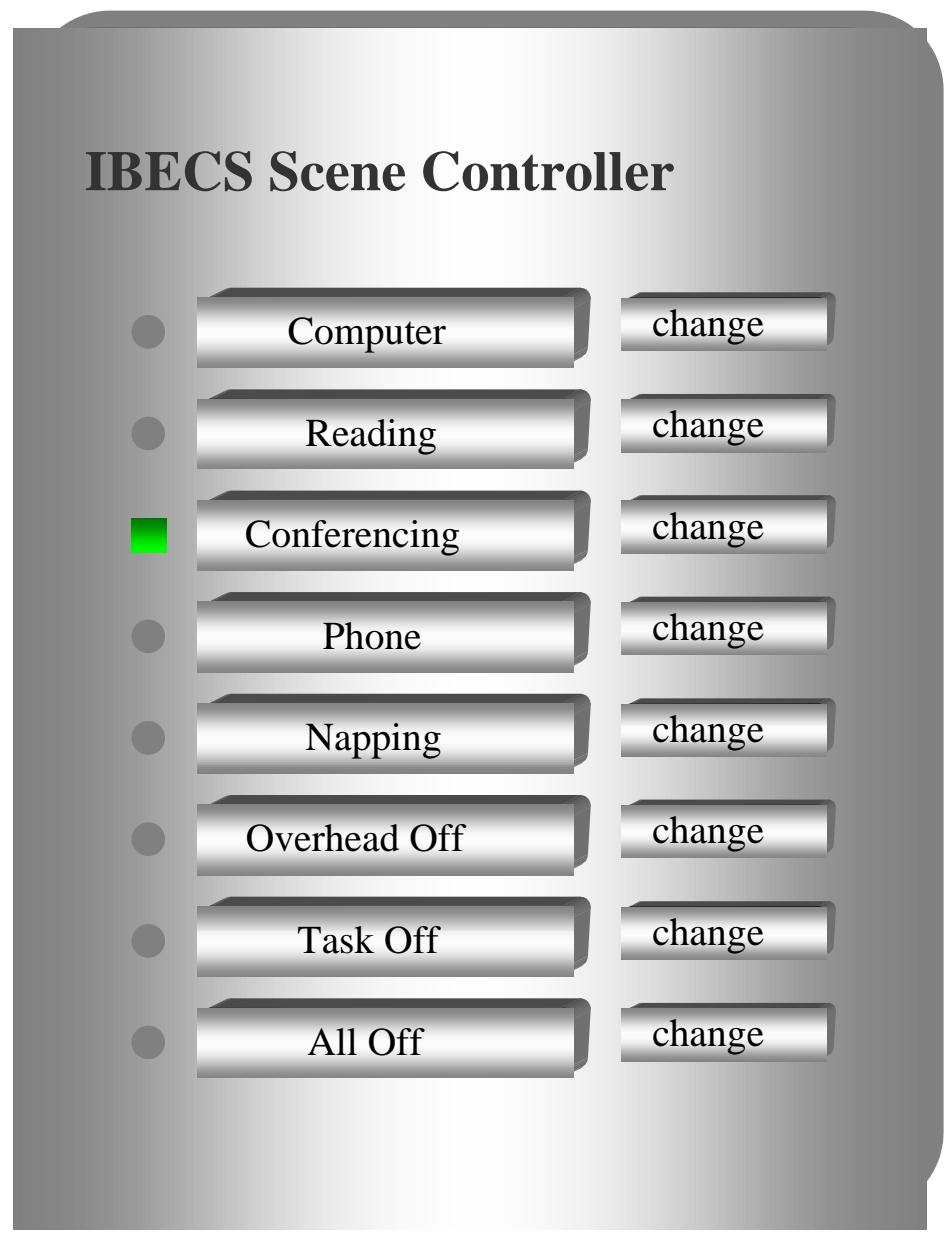

Artist's conception of what the IBECS Scene Controller will look like after adding graphics and annunciators.

\section{Integration of Control Interface into Task Lamp Fixtures}

This subsection involved the development of an improved control interface for integration into portable task lamp fixtures that incorporate Personal Workspace Control (PWC) modifications.

Several IBECS-controlled task lamp modifications that Vistron had previously delivered to LBNL successfully confirmed the concept of PWC. However, the implementation required customization to fit within the available space within the unique geometry of each type of commercial fixture. It was concluded that a new, more universal, alternative arrangement needed to be devised. What evolved was a scheme that moved the control electronics to a plug-in module at the end of the fixture's power cord, much like the ubiquitous "wall-wart” commonly used with consumer electronics. This configuration also was adjudged to be more acceptable to a typical user. In the earlier version, two cords had to be run into the lamp base: one for power and one for network control. This proved to be somewhat cumbersome and unsightly. With the new arrangement the IBECS network connection was routed to a modular connector on case of the plug-in 
interface module. This resulted in a more esthetically acceptable single consolidated power/control cable connecting with the base of the lamp.

As in the previous version, 3-step dimming was utilized. Commercial lamp fixtures with this function were found to be generally available utilizing either spiral smaller CFL (12/18/29 watts) or higher output Circleline ${ }^{\circledR}$ lamps. We chose to utilize the latter option for this demonstration project.

Also, maintaining a design requirement of the earlier versions, we implemented a dualmode control arrangement. The dimming level is normally controlled via the IBECS network, but reverts to manual control from a switch on the lamp in the event that the network is not functioning. The prior versions used a conventional 3-way rotary switch mounted in the base or stem of the fixture for this control. The new control arrangement replaces that control with a miniature, momentary, Up/Down toggle switch. The use of a toggle switch allows the lamp to revert to off when bus control is lost, rather than to the last rotary switch position, which is likely irrelevant.

It was determined that this new control interface logic would be implemented with an embedded microprocessor with "Flash" program storage. This provided the ability to easily modify the control parameters as we experimented with typical user preferences. A productized version of this controller would likely use either a lower-cost gate array or a permanently programmed microprocessor.

\section{Implementation}

Both lamps selected for modification on this subsection were manufactured by Technical Consumer Products (TCP), 300 Lena Drive, Aurora, OH 44202. TCP has a wide national distribution network; we elected to purchase our samples via the Internet from GoodMart (www.goodmart.com), a national distributor that is located at 45 Main Street, Suite \#403, Brooklyn, NY 11201.

The floor lamp that we selected is the TCP Model 53065BS, identified as a 3-way brushed steel fluorescent Torchiere lamp. Reproduction of the TCP catalog pages for this lamp appears below. 

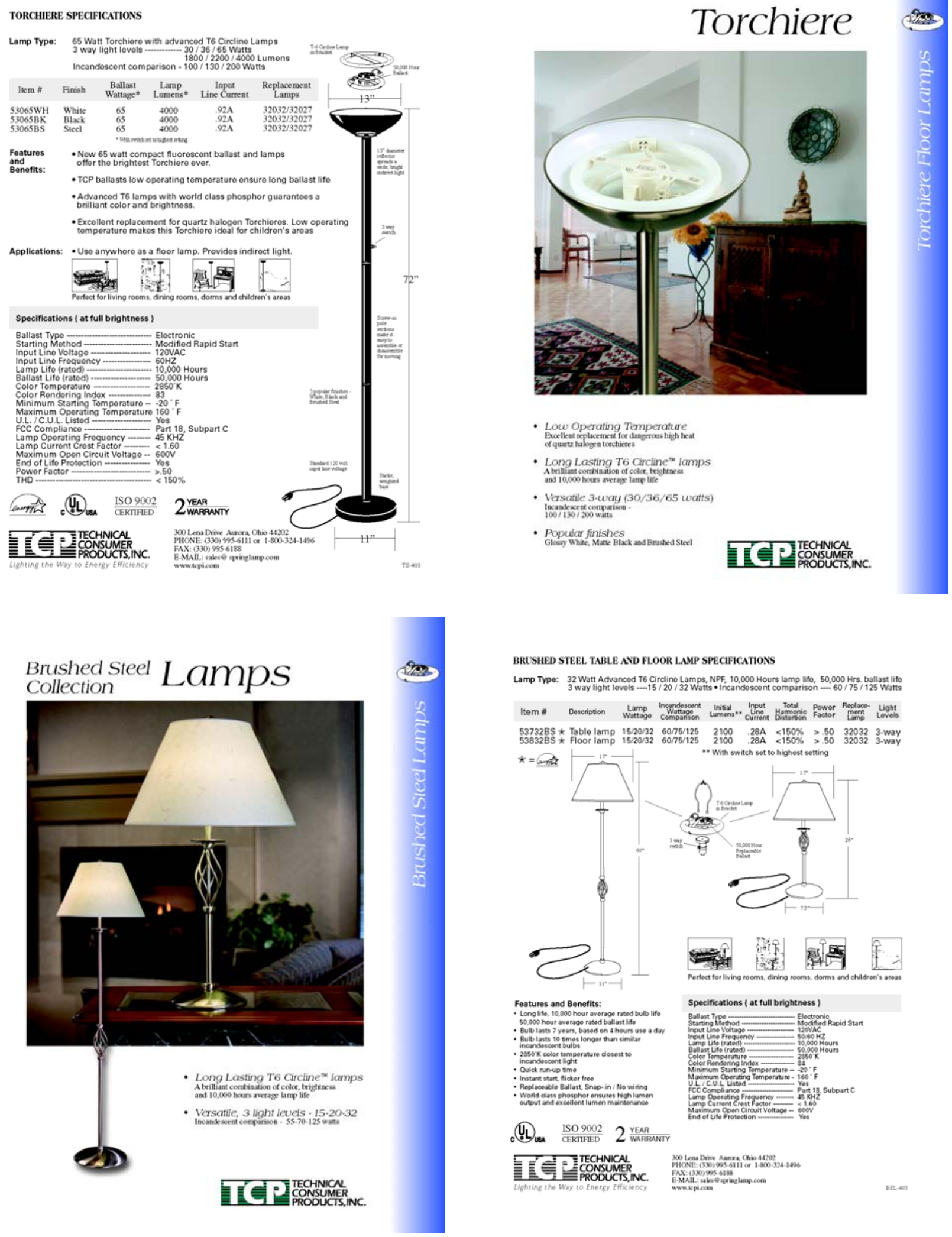

BRTSHED STEEL TABLE AND FLOOR LAMP SPECIFICATIONS

$\begin{array}{ll}\text { Lamp Type: } & 32 \text { Watt Advancod T6 Circline Lamps, NPF, 10,000 Hours lamp lito, } 50,000 \text { Hrs ballast life } \\ 3 \text { way light lovols }-15 / 20 / 32 \text { Watts } & \text { Incandoscont comparison }-60 / 75 / 125 \text { Watts }\end{array}$

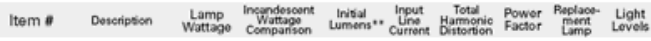
$\begin{array}{lllllllll}53732 B S \star \text { Table lamp } & 15 / 20 / 32 & 6077 / 125 & 2100 & .28 \mathrm{~A} & <150 \% & >50 & 32032 & \text { 3-way } \\ 53832 \mathrm{BS} * \text { Floor lamp } & 15 / 20 / 32 & 60 / 75 / 125 & 2100 & .28 \mathrm{~A} & <150 \% & >.50 & 32032 & \text { 3-way }\end{array}$

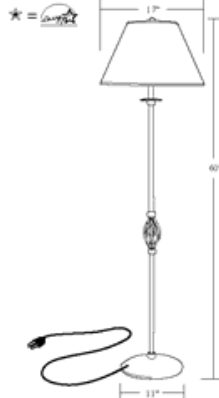

Features and Benefits:

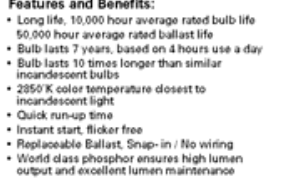

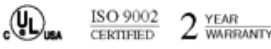
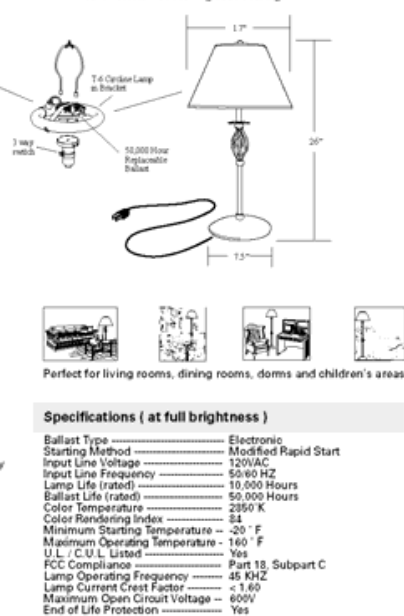

ED ETEHNCA

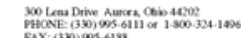

TCP catalog pages showing torchiere floor lamp selected for modification. 
The TCP Torchiere includes a dedicated ballast having three hard-wired line voltage connections: Common (Neutral), Line Low and Line High. It is supplied with a 3-way rotary canopy switch that is mounted in the upright staff of the fixture. The unmodified lamp has a specified consumption of 30/36/c65 watts with an output of 4000 Lumens at maximum intensity.

The table lamp that we selected is the TCP Model 53732BS, identified as a 3-way brushed steel fluorescent table lamp. Reproduction of the TCP catalog pages for this lamp appears below.

The table lamp utilizes a CFL-style combined ballast and lamp with an Edison 3-way screw-in base. The lamp is supplied with a 3-way socket having an integral rotating key switch for dimming control. (Note that also TCP offers a floor lamp in the same style as the table lamp. Presumably the same modifications used for the table lamp would be applicable to this version). Converting this lamp to network control necessitated the replacement of the original 3-way keyed socket to a non-keyed version. This was easily accomplished by unscrewing its threaded base and replacing the whole socket assembly with a keyless 3-way socket with a metal shell (Leviton P/N 7065).

The table lamp is designed to be used with a screw-in (Edison E-26) T-6 Circline ${ }^{\mathrm{TM}}$ electronic ballast/bulb adaptor (TCP \#16550L). It has a specified 10/20/32 watt input, a $2850 \mathrm{~K}$. color temperature, a CRI of 84 and a 2100 lumen initial output. It has a 10,000 hour life rating and includes end-of-life protection. It utilizes a modified programmed instant start, has a $<1.6$ Crest Factor and operates at approximately $45 \mathrm{kHz}$

\section{Lamp Modifications}

\section{Torchiere}

As supplied, the Torchiere is broken down into interconnected sections with threaded joints. During assembly of these components, the lamp's original canopy dimming switch was replaced with a miniature momentary toggle switch (DigiKey P/N EG2392). The original line cord wiring was removed and replaced by a 6-conductor cable (DigiKey A124-X-ND) with a nominal length of 12 feet. This cable exits the fixture via the same groument in the lamp base that was previously occupied by the line cord.

\section{Table Lamp}

After replacing the keyed Edison socket with a non-keyed (e.g. no dimming switch) version, it was necessary to drill a hole in the base of the fixture to hold the same type of dimming control toggle switch as was used above. Also, the line cord wiring was replaced using the same type of 6-conductor cable that was previously used with the Torchiere. Again, this cable exits the fixture via the groument that was previously used by the former line cord. 


\section{Control Interface Packaging}

The control electronics were housed in a commercially available vented enclosure with an integral 3-prong power plug. This case, as supplied, is pictured on the left. It includes a notched outlet for a connecting cable and is supplied with a rectangular molded strain relief for the cable entry. This case is molded from black G. E. Noryl plastic and has outside dimensions of 3.34"H x 2.70"W x 2.26" D. It is distributed by Jameco http://www.jameco.com/ as their P/N 115043. A $15 \mathrm{~cm}$ (w) x $19 \mathrm{~cm}$ (h) rectangular hole was cut in the center of the top of the case in order to mount a RJ45/6-4 female with bezel connector (DigiKey P/N H9062) that mates with a plug-in cable that serves as the IBECS network control connection.

The drawing to the right illustrates the geometry of the perforated prototyping circuit board that is shaped to fit in the enclosure. It was cut from epoxy material with plated through holes and was purchased from Jameco as their P/N 37621.

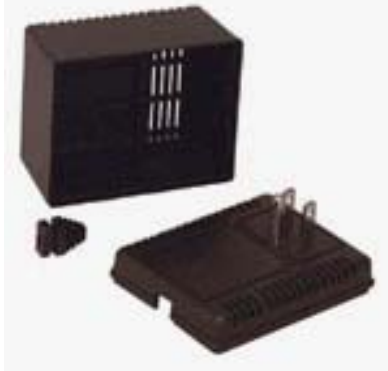

Case used to house control electronics

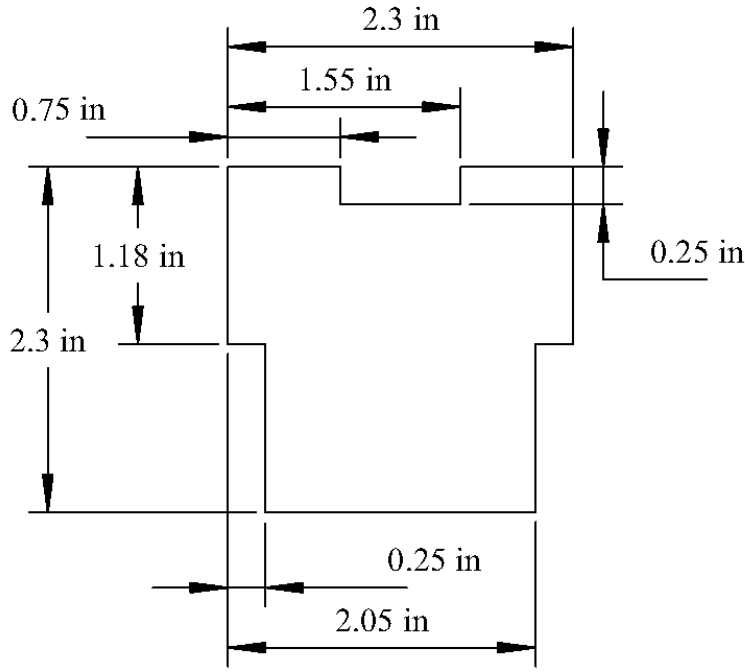

Geometry of perforated prototyping circuit board 


\section{Interface Control Circuitry}

The control circuitry, 1-Wire network and isolated off-line power supply module were all mounted on the prototyping board. The assembled prototyping board was secured to the bottom of the enclosure nesting next to the ends of the AC plug prongs with hot melt glue. The six-conductor cable connecting to the lamp carries switched AC power to energize the desired tube(s) as well as the control signals from the toggle switches mounted on the lamps. The six conductors in the cable were each soldered to terminals on the prototyping board. The cable was then routed through the snap-in strain relief that is supplied with the enclosure. The RJ-11 jack (J2) with its snap-on bezel is secured in the cutout in the top of the enclosure with ACC (superglue) adhesive. The two portions of the case are secured together with superglue.
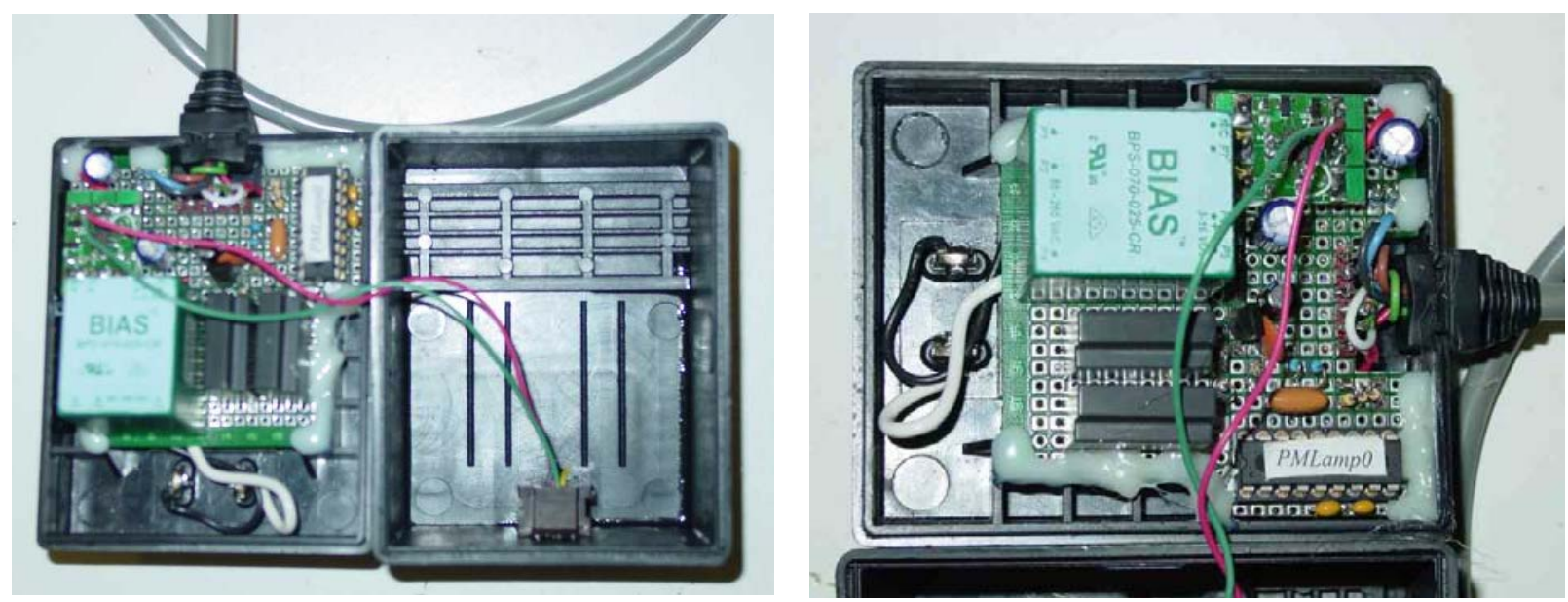

Images of the control interface circuitry

The schematic diagrams that follow show the system electrical components as well as the devices on the prototyping board. Components that are located in the lamps are so noted. There are individual schematics for both the Floor Lamp and the Table Lamp versions. A consolidated Bill of Materials applies to both versions. A portion of the circuitry is contained on a modified 1-Wire control daughter card, the D2P Module, purchased from Embedded Data Systems http://www.embeddeddatasystems.com/. 


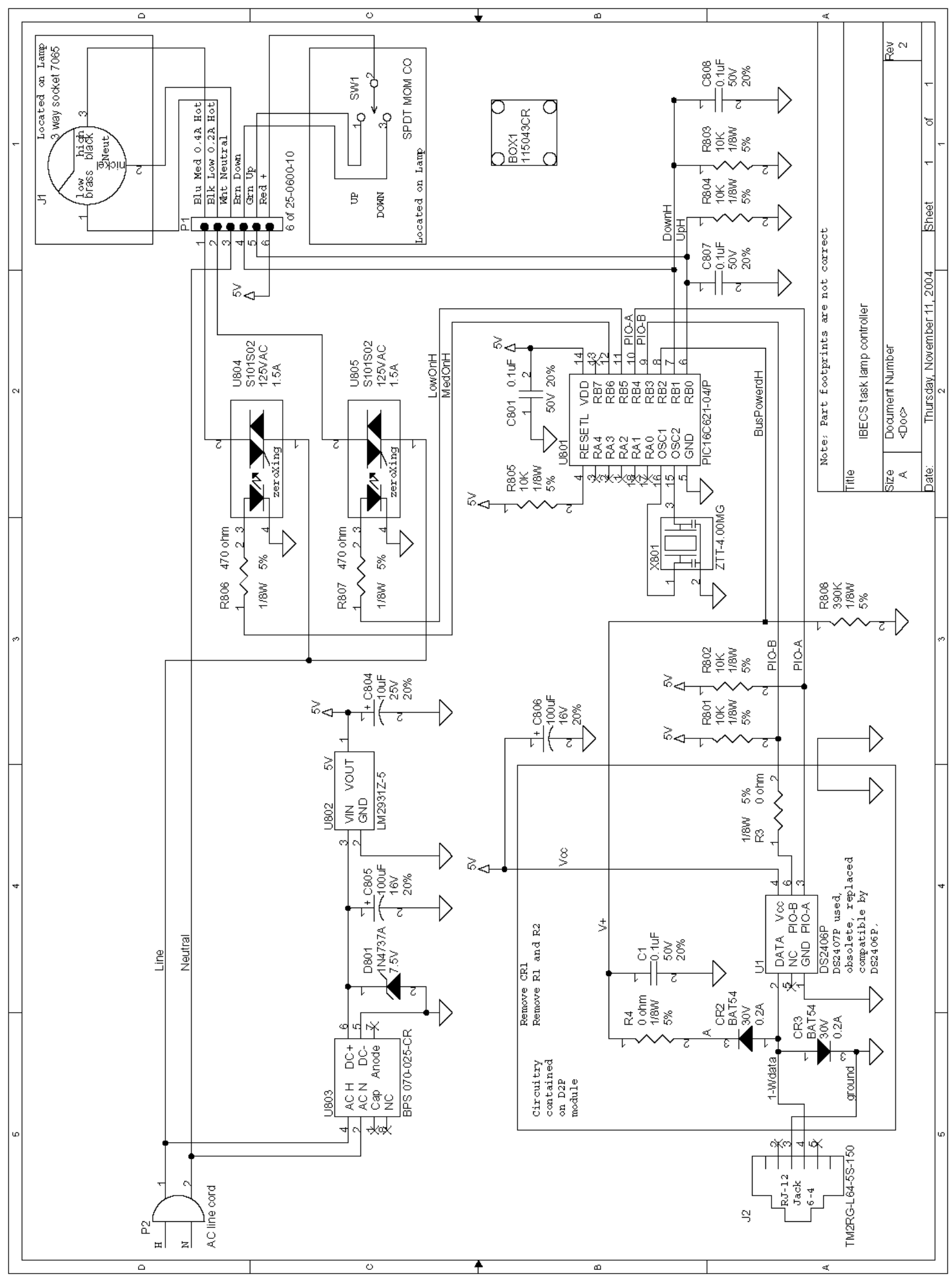

Schematic diagram of IBECS task lamp controller 


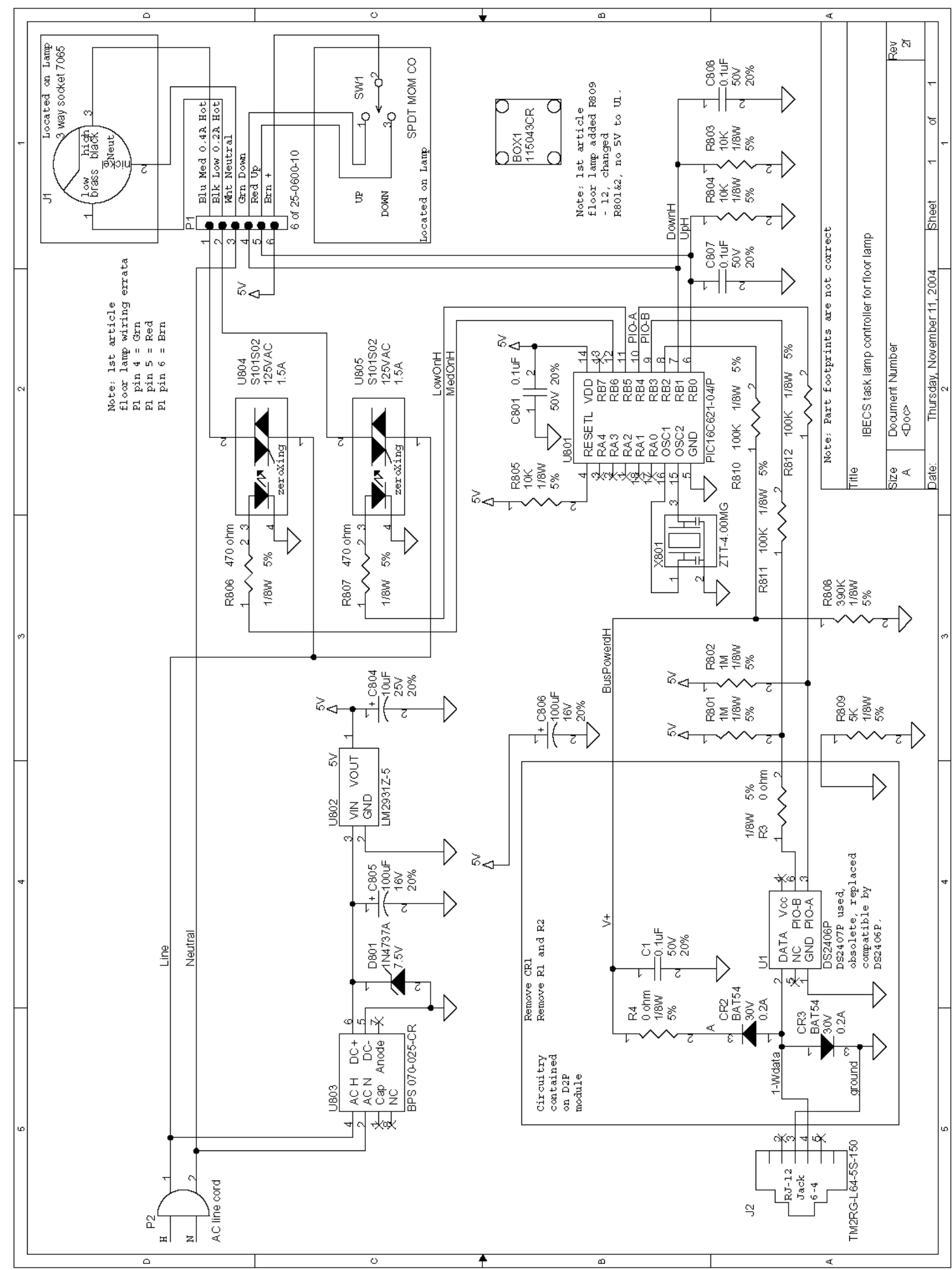

Schematic diagram of IBECS controller for floor lamp 
Bill of Materials for IBECS Task Lamp Controller

Revision: 3, 12/13/04

\begin{tabular}{|c|c|c|c|c|}
\hline Qty & Ref & Mfg \& P/N & Dist , P/N, Comment & Value \\
\hline 1 & BOX1 & GPB483 & Jameco $115043 C R$ & 115043CR \\
\hline 2 & CR2 & Zetex BAT54STA & DigiKey BAT54SCT-ND & BAT54 \\
\hline & CR3 & Zetex BAT54STA & DigiKey BAT54SCT-ND & BAT54 \\
\hline 4 & C1,C801,C807,C808 & $0805,20 \%$ & Generic & $0.1 \mathrm{uF} / 50 \mathrm{~V}$ \\
\hline 1 & C804 & $20 \%$ & generic & $10 \mathrm{uF} / 25 \mathrm{~V}$ \\
\hline 2 & $\mathrm{C} 805, \mathrm{C} 806$ & $20 \%$ & Generic & 100uF/16V \\
\hline 1 & D801 & Diodes Inc. 1N4737A-T & $\begin{array}{l}\text { DigiKey 1N4737ADITR- } \\
\text { ND }\end{array}$ & 1N4737A \\
\hline 1 & $\mathrm{D} 2 \mathrm{P}$ & $\begin{array}{l}\text { Embedded Data } \\
\text { Systems }\end{array}$ & Requires modification & \\
\hline 1 & J1 & Leviton \#7065 & Replace on Lamp & 3 way socket $\mathbf{1}$ \\
\hline 1 & $\mathrm{~J} 2$ & $\begin{array}{l}\text { Hirose TM2RG-L64-5S- } \\
150\end{array}$ & DigiKey H9062-ND & TM2RG-L64-5S-150 \\
\hline $1 / 4$ & P1 & Aries $25-0600-10$ & DigiKey A115-ND & $1 / 4 \quad 25-0600-10$ \\
\hline 1 & $\mathrm{P} 2$ & Alpha 1176C-X & DigiKey A124-X-ND & $\begin{array}{l}\text { 12' Multi-Conductor \#22 } \\
\text { Cable }\end{array}$ \\
\hline 2 & R3,R4 & $0805,5 \%$ & Generic & $0 \mathrm{ohm}$ \\
\hline 5 & $\mathrm{R} 801,802,803,804,805$ & $0805,5 \%$ & Generic & $10 \mathrm{~K}$ \\
\hline 2 & R806,807 & $0805,5 \%$ & Generic & 470 ohm \\
\hline 1 & R808 & $0805,5 \%$ & Generic & $390 \mathrm{~K}$ \\
\hline 1 & SW1 & E-Switch EG2392 & Mounted on Lamp & SPDT MOM CO \\
\hline 1 & U1 & Dallas DS2406P & Dallas/Maxim & $\mathrm{N} / \mathrm{A}$ \\
\hline 1 & U801 & $\begin{array}{l}\text { Microchip PIC16C621- } \\
\text { 04/P }\end{array}$ & $\begin{array}{l}\text { Microchip PIC16C621- } \\
\text { 04/P }\end{array}$ & $\mathrm{N} / \mathrm{A}$ \\
\hline 1 & U802 & National LM2931Z-5 & LM2931Z-5.0NS-ND & LM2931Z-5 \\
\hline 1 & U803 & BIAS BPS 070-025-CR & DigiKey 440-1000-ND & 3-16 v@1/4 w \\
\hline 2 & U804,805 & Sharp S101S02 & Jameco 164988 & S101S02 \\
\hline 1 & $\mathrm{X} 801$ & ECS ZTT-4.00MG & Digi-Key X902-ND & ZTT-4.00MG \\
\hline
\end{tabular}

Notes : 1 - Only applicable to the Table Lamp version

\section{Lamp Control User Interface}

The Task Lamp Dimming level can be controlled by either via commands sent over the network from the control console or from the local momentary toggle switches mounted on the lamps, in the event the network is inoperative.

\section{Network Lamps Control}

The lamp operates under 1-Wire control whenever the controller is connected to a powered network. The lamp appears to the 1-Wire network discovery process as a DS2407P dual switch, which is eclectically compatible with the Dallas/Maxim DS2406P chip. This 1-wire IC has two ports designated as PIO-A and PIO-B. In this application they are both configured as digital outputs. Each of these output lines is connected to the embedded PIC microprocessor. When network control is operative, the following Truth Table defines the relationship between the lamp's dimming level and the DS2407 output state combinations: 


\begin{tabular}{|l|l|l|l|l|}
\hline Dimming Level - & Off & Low & Med & High \\
\hline PIO-A - & 1 & 1 & 0 & 0 \\
\hline PIO-B - & 1 & 0 & 1 & 0 \\
\hline
\end{tabular}

Note that the legacy 1-Wire network is noise sensitive and consequently, the network cabling should be routed in such a way as to avoid coupling with of power line induced noise. It is suggested that future versions of the controller incorporate the newly developed SlimNet differential network interface. The greatly improved common mode rejection performance inherent to SlimNet would eliminate such complications with power line induced interference.

\section{Manual Dimming Control}

The switches on the task lamps are operative when the controller network interface senses that the 1-Wire bus is not powered. Some USB port adaptors put the 1-Wire bus in a powered state, whenever the controlling PC is turned on, but during intervals when the control program is not in operation. For this reason, the use of RS232 adaptors such as the Dallas 9097U or the iButtonLink (which do not have this anomaly) are recommended. It is suggested that future versions of the controller incorporate an enhanced protocol that instead of checking for voltage on the network, that the test instead be based on the presence of data on the network. This enhancement would permit the controller to operate correctly with all types of commercial port adaptors.

When under manual dimming control, briefly depressing the switches upward increases the light level, while a downward depression decreases the light level. Holding the switches up or down causes auto repeat at a rate of approximately once per 1 second.

\section{Microprocessor Program}

U801 is a flash-programmed microprocessor. Its custom code permits it to serve as a state machine to coordinate the lamp dimming settings in response to various combinations of network control and local switch operations. The code is written in PIC BASIC, as follows:

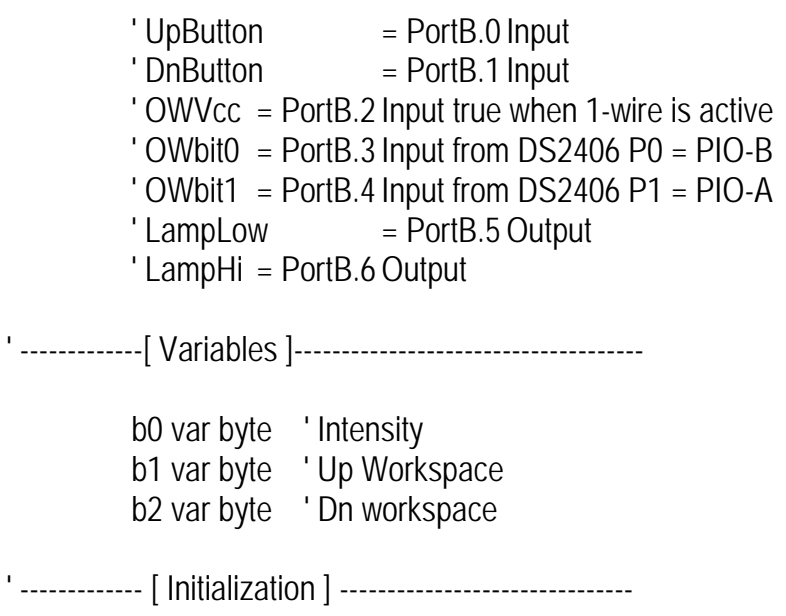




$\begin{array}{ll}\text { TRISB = \%10011111 } & \text { ' Set Rb5 \& Rb6 as outputs } \\ \text { b0 }=0 & \text { ' Start in OFF state } \\ \text { b1 }=0 & \text { ' Clear Button workspaces } \\ \text { b2 }=0 & \text { ' Ditto }\end{array}$

'[ Main Code ]

Main:

$$
\begin{array}{ll}
\text { portB. } 5=\text { b0. } & =\text { portB. } 3{ }^{\prime} \text { LSB Lamp Control }=\text { LOW } \\
\text { portB. } 6=\text { b0.1 } & =\text { portB. } 4 \text { ' MSB Lamp Control }=\text { MED }
\end{array}
$$

If portB. $2=1$ Then PC ' Network is up, so use 1-wire control

Button PortB.0,0,50,50,b1,1,Up

Button PortB.1,0,50,50,b2,1,Dn

Pause 20

Goto Main

' ----------- [ Subroutines ]

Up: If $\mathrm{b} 0<>3$ Then $\mathrm{b} 0=\mathrm{b} 0+1$ ' $\mathrm{OK}$ to increment intensity level Goto Main

Dn: If $\mathrm{b} 0<>0$ Then $\mathrm{b} 0=\mathrm{b} 0-1 \quad$ 'Decrement intensity if in valid range Goto main

PC:
b0.0 $=$ portB. 3
b0.1 $=$ portB. 4
' Transfer OW LSB to LampLow
Goto Main
' Transfer OW LSB to LampHi 